$\begin{gathered}\text { Revista do Departamento de Geografia } \\ \text { Universidade de São Paulo } \\ \text { www.revistas.usp.br/rdg }\end{gathered}$
Volume Especial - XVII SBGFA / I CNGF (2017)

\title{
Condicionantes Morfológicos e Geológicos dos Escorregamentos Rasos na Bacia do Rio Santo Antônio, Caraguatatuba/SP
}

\section{Morphological and Geological Conditioning Factors on Shallow Landslides in Santo Antonio Basin, Caraguatatuba/SP}

\author{
Helen Cristina Dias \\ Universidade de São Paulo \\ helen.dias@usp.br \\ Vivian Cristina Dias \\ Universidade de São Paulo \\ vivian.cristina.dias@usp.br \\ Bianca Carvalho Vieira \\ Universidade de São Paulo \\ biancacv@usp.br
}

Resumo: O objetivo deste artigo foi analisar a influência dos parâmetros morfológicos e geológicos na ocorrência de escorregamentos na bacia hidrográfica do Santo Antônio, intensamente afetada por escorregamentos e corridas de detritos durante o verão de 1966/1967. Para a confecção dos mapas morfológicos e geológicos, foram utilizados respectivamente, um Modelo Digital de Elevação (MDE) obtido do sistema LiDAR, e o mapa geológico digitalizado em escala 1:50.000. A partir disso, foi realizada uma análise integrada dos parâmetros em conjunto com as cicatrizes de escorregamentos, mapeadas a partir de fotografias aéreas e ortofotos de 1973. Os resultados mostraram que os parâmetros indicaram maior suscetibilidade a escorregamentos nas encostas côncavas com inclinação $>30^{\circ}$. Desta maneira, foi possível verificar as classes preferenciais para ocorrência de escorregamentos na bacia do Santo Antônio, sendo este um fator essencial para futuros mapeamentos de áreas de risco no município.

Palavras-chave: Bacia Hidrográfica; Morfologia; Geologia; Serra do Mar.

\begin{abstract}
The aim of this paper was to analyze the influence of morphological and geological parameters on the landslides occurrence on Santo Antônio basin, which was highly affected by landslides and debris flow in 1966/1967 summer season. The production of morphological and geological maps were based on a high-resolution $\left(5 \mathrm{~m}^{2}\right)$ digital elevation model (DEM) and a detailed geological map of Mineral Resources Research. From this, it was made an evaluation based on analysis of an agreement between predicted susceptibility and the landslide scar's map from aerial photographs and orthophotos from 1973. The results showed concave slopes and angles $>30^{\circ}$ as the most susceptible areas. Thus, it is possible verify preferred classes to landslide occurrence on Santo Antônio basin, an important factor to future risk mapping of the area.
\end{abstract}

Keywords: Basin; Morphology; Geology; Serra do Mar. 


\section{INTRODUÇÃO}

Os escorregamentos são processos naturais recorrentes em ambientes com encostas declivosas submetidas à elevados e concentrados volumes pluviométricos, como a Serra do Mar. Esta se apresenta como escarpa de falha localizada no litoral das regiões sul e sudeste do Brasil, com origem em processos tectônicos provenientes do Cenozóico, com grande diversidade de embasamentos litológicos, tais como associações migmatíticas e metamórficas e diversos complexos ígneos (ALMEIDA E CARNEIRO, 1998). Há ainda muitas falhas e fraturas com orientação geral NE-SW, que revelam um alto controle estrutural e grandes áreas potencialmente instáveis a escorregamentos (CRUZ, 1974).

Características morfológicas como curvatura e ângulo das encostas associadas aos parâmetros litológicos e estruturais podem contribuir para a identificação de classes mais propícias à ocorrência de processos morfodinâmicos como escorregamentos, permitindo inferir os fatores condicionantes dos mesmos (SIDLE et al.; 1985; PACHAURI E PANT, 1992; VIEIRA et al., 2010). Desta maneira, o objetivo deste trabalho foi analisar a influência dos parâmetros geológicos e morfológicos na ocorrência de escorregamentos na bacia do rio Santo Antônio no município de Caraguatatuba / SP.

\section{ÁREA DE ESTUDO}

O município de Caraguatatuba está localizado no litoral norte do Estado de São Paulo (Figura 1), se estendendo desde a planície litorânea até as altas elevações da Serra do Mar. Este ambiente apresenta um destacado histórico de eventos morfodinâmicos relacionados à ocorrência de escorregamentos e corridas de detritos, razão pela qual a área foi escolhida para estudo.

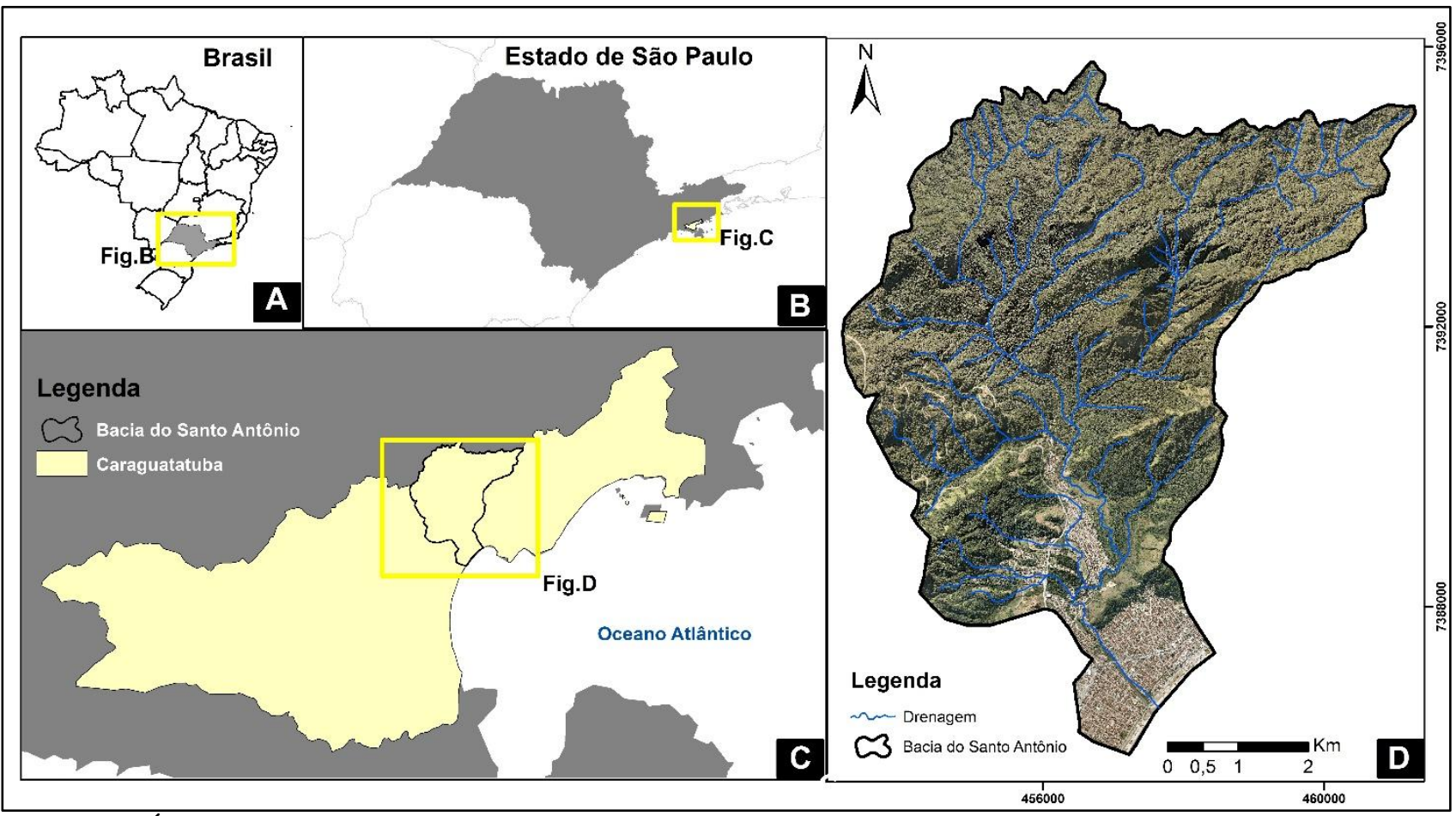

Figura 1: Área de estudo - A: São Paulo em relação ao Brasil; B: Caraguatatuba em relação a São Paulo; C: Município de Caraguatatuba; e D: bacia do rio Santo Antonio.

O evento mais marcante do município ocorreu em março de 1967 no qual foram registrados escorregamentos e corridas de detritos generalizados, com sua deflagração associada a um evento climático intenso, com precipitação de cerca de $586 \mathrm{~mm}$ em apenas $48 \mathrm{hs}$, valor este acima da média da região (CRUZ, 1974). Na ocasião milhares de pessoas foram afetadas, inclusive com registro de mortos e grande número de desabrigados, além da destruição da infraestrutura coletiva, falta de comunicação e energia elétrica, acarretando em prejuízos à economia local (Figura 2). 
Uma das áreas marcadamente afetadas foi a bacia do Santo Antônio (BSA), selecionada para estudo diante o registro de escorregamentos de alta magnitude no citado evento de 1967.

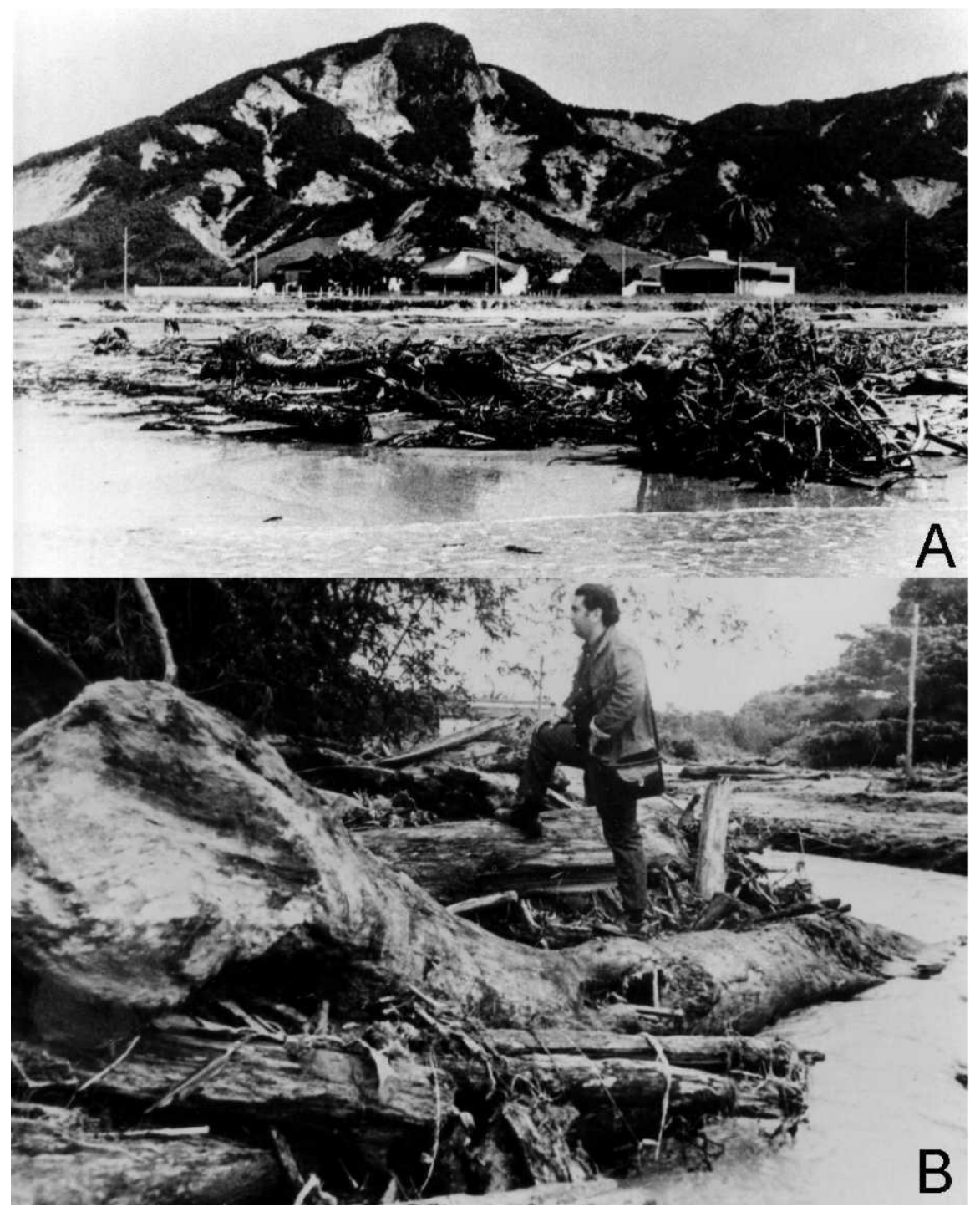

Figura 2: (A) Escorregamentos no Morro do Jaraguá; (B): Depósitos das corridas de detritos em Caraguatatuba, (SP) 1967. Fonte: Arquivo Público de Caraguatatuba.

\section{MATERIAIS E MÉTODOS}

Para alcançar o objetivo proposto foram elaborados mapas morfológicos de Curvatura e Ângulo de encostas, utilizando o Modelo Digital de Elevação, resolução de $25 \mathrm{~m}$, gerado a partir de dados LiDAR (Light Detection and Ranging) da Empresa Paulista de Planejamento Metropolitano S/A - EMPLASA (Contrato de Licença de uso CLU $N^{o}$ 049/14). O mapa geológico de escala 1:50.000 foi digitalizado a partir da carta de Caraguatatuba (SF-23-Y-D-VI-1) da Companhia de Pesquisa de Recursos Minerais (CPRM).

Para o mapeamento das cicatrizes dos escorregamentos foram utilizados ortofotos (resolução de $1 \mathrm{~m}$ ) do Instituto Florestal, de 2000, o trabalho de Fúlfaro et al. (1976) e fotografias aéreas de 1973 na escala 1:20.000. A avaliação conjunta da influência dos parâmetros geológicos e morfológicos foi realizada com base no método proposto por Gao (1993), que faz uso de três índices: Potencial de Escorregamentos (PE): razão entre o número de células de cada classe afetada pelas cicatrizes e o total de células dessa mesma classe; Concentração de cicatrizes (CC): razão entre o número de células de cada classe afetada pelas cicatrizes e o valor total de células afetadas por escorregamentos na bacia e a Frequência $(F)$ : razão entre o número de células de cada classe e o total de células da bacia. 


\section{RESULTADOS E DISCUSSÕES}

A partir da correlação do mapa de cicatrizes com os mapas morfológicos e geológicos foi possível avaliar quais as classes mais afetadas pelos escorregamentos.

Com base nos índices de Frequência (F), de Concentração de Cicatrizes (CC) e de Potencial de Escorregamento (PE), foi possível averiguar que muito embora os Granitóides Caraguatatuba sejam o litotipo mais abundante na bacia e consequentemente com maior concentração dos processos de escorregamentos (81,5\%), são na verdade, as Rochas Parametamórficas que apresentaram um PE maior para a ocorrência do processo, com 10,4\% (Figura 3).

Foi possível observar também que há uma vasta quantidade de falhas e fraturas na bacia, o que indica um grande controle estrutural, uma vez que ela é cortada por extensas descontinuidades que atuam como caminhos preferenciais de alteração, afetando a dinâmica hidrológica dos fluxos subterrâneos (FERNANDES E AMARAL, 1996; HART, 2007). Em especial, a presença da falha de Caraguatatuba no quartzito, sob intensos níveis de intemperismo, poderá dar origem a um material mais arenoso (FERREIRA, 2013), e consequentemente, inconsolidado, friável e mais instável a processos morfodinâmicos.

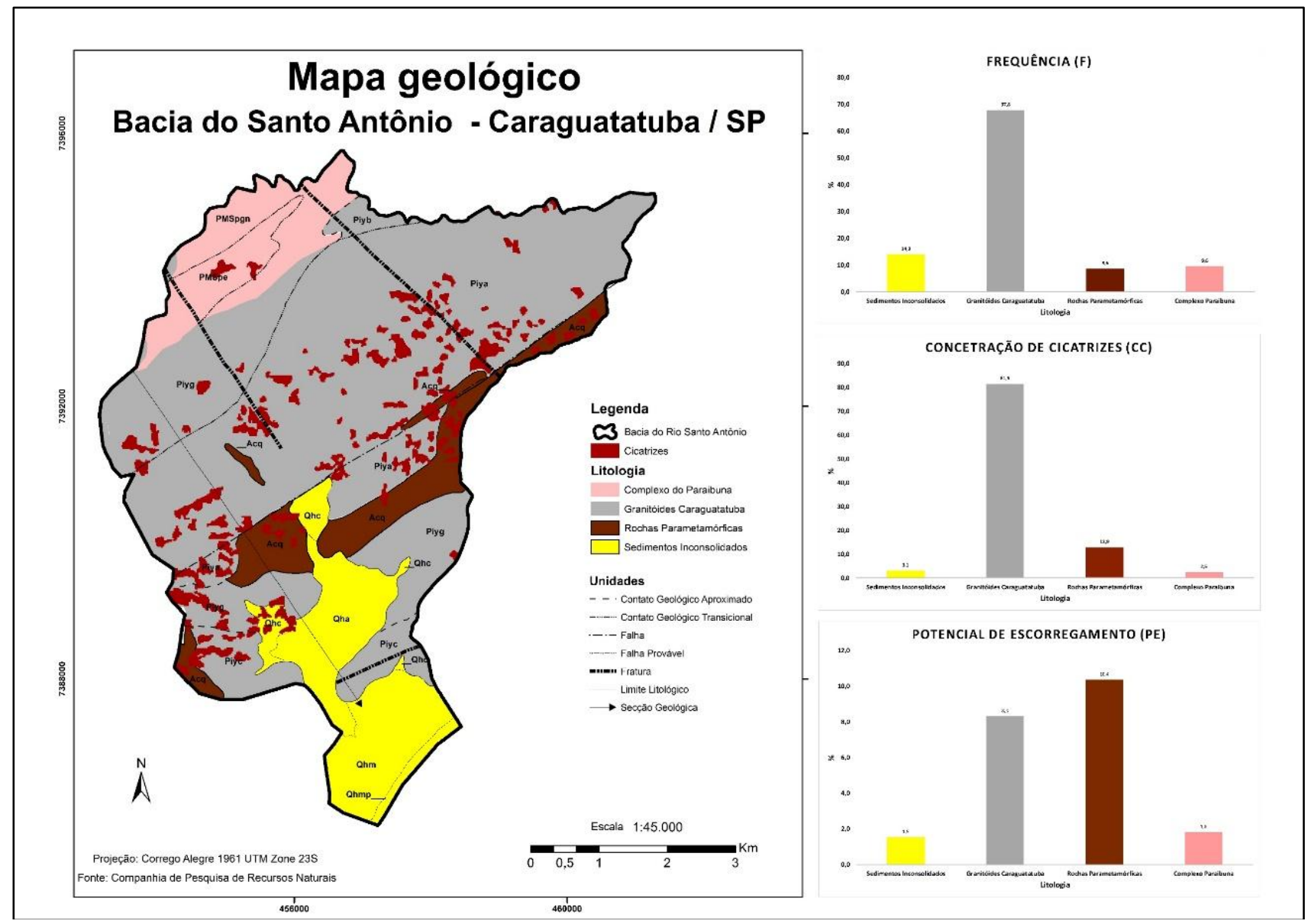

Figura 3: Geologia da BSA, onde se verifica o predomínio dos Granitóides Caraguatatuba e uma grande presença de falhas e fraturas na bacia.

O parâmetro morfológico de Ângulo de Encosta (Figura 4), analisado a partir dos índices de CC e PE, possibilitou estabelecer a classe de ângulo na qual processos de escorregamentos foram mais recorrentes. Assim, de acordo com o índice de CC, os escorregamentos se concentraram nas classes entre $20,1^{\circ}$ a $30^{\circ}$ $(33,1 \%)$, embora o índice PE tenha mostrado que o intervalo com maior ocorrência de cicatrizes tenha sido em encostas com ângulos $>30^{\circ}$. Outros autores como Lan et al. (2004), Vieira e Ramos (2015) e Nery e Vieira (2014), identificaram, respectivamente, valores similares com intervalos de $30^{\circ}-50^{\circ} ; 30^{\circ}-40^{\circ}$ e $30^{\circ}-50^{\circ}$ como preferenciais para a ocorrência do processo. Vieira e Ramos (2015) e Nery e Vieira (2014) fizeram suas ponderações no mesmo ambiente da BSA, na Serra do Mar, o que demonstra que tais inferências podem ser uma tendência regional. 


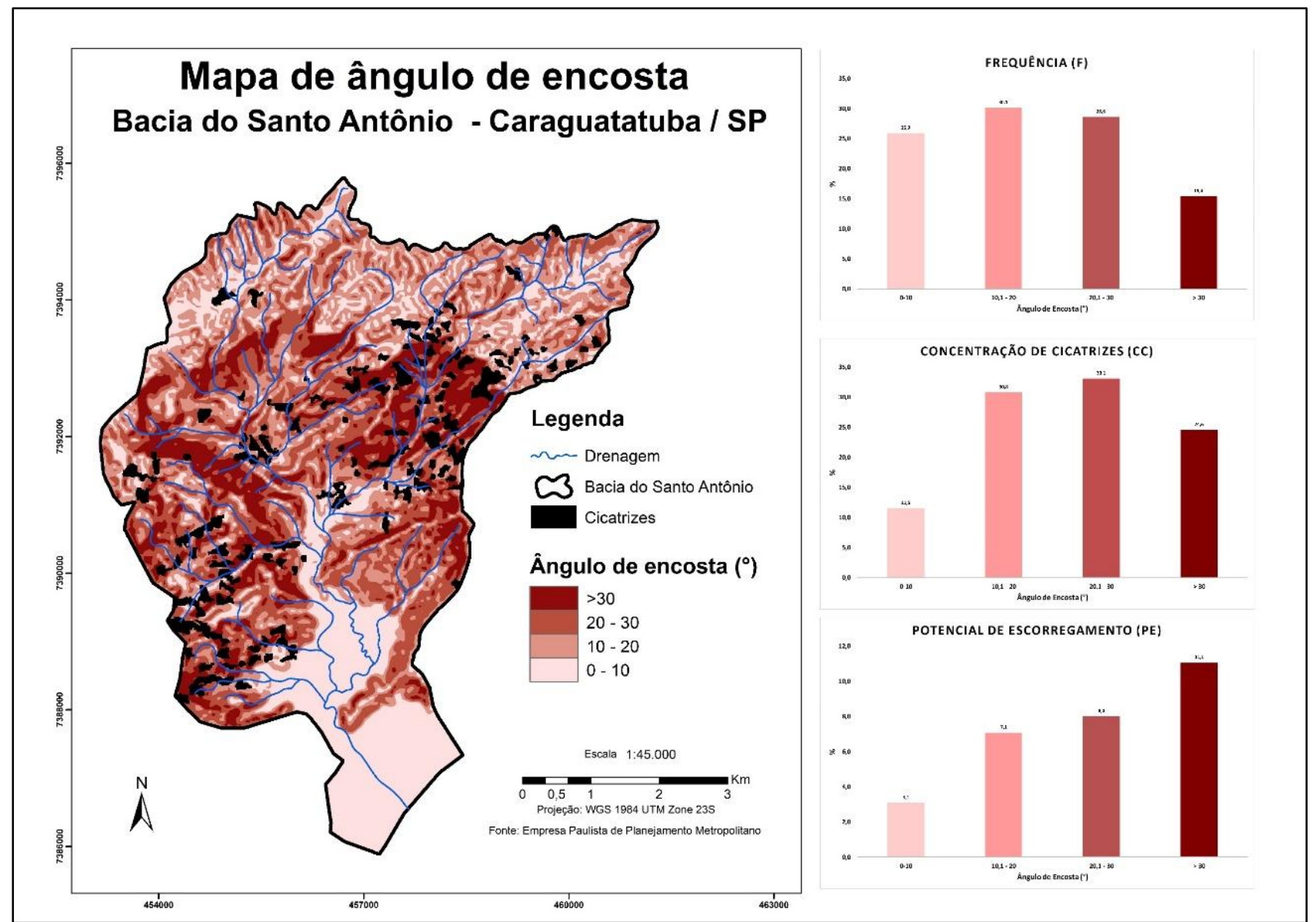

Figura 4: Ângulo de encosta da BSA, onde se verifica uma concentração de ângulos $>30^{\circ}$ na porção central da bacia, onde também se concentram as cicatrizes de escorregamentos.

O segundo parâmetro morfológico analisado foi o de curvatura (Figura 5), no qual se verificou que a feição com maior potencial de escorregamento (PE) foi a côncava $(8,1 \%)$, cuja característica é a tendência a concentrar fluxos facilitando a ocorrência de escorregamentos (MONTGOMERY e DIETRICH, 1994). Tal resultado é similar ao encontrado por Fernandes et al. (2001), que estudaram os escorregamentos que ocorreram nas bacias de Quitite e Papagaio no evento de 1996, onde diagnosticaram um alto controle da morfologia da área na ocorrência e localização das cicatrizes de escorregamentos. Lan et al. (2004) por sua vez, desenvolvem suas análises na China e apresentam valores condizentes com a realidade da Serra do Mar, embora algumas condições morfoclimáticas possam se mostrar diferentes. 


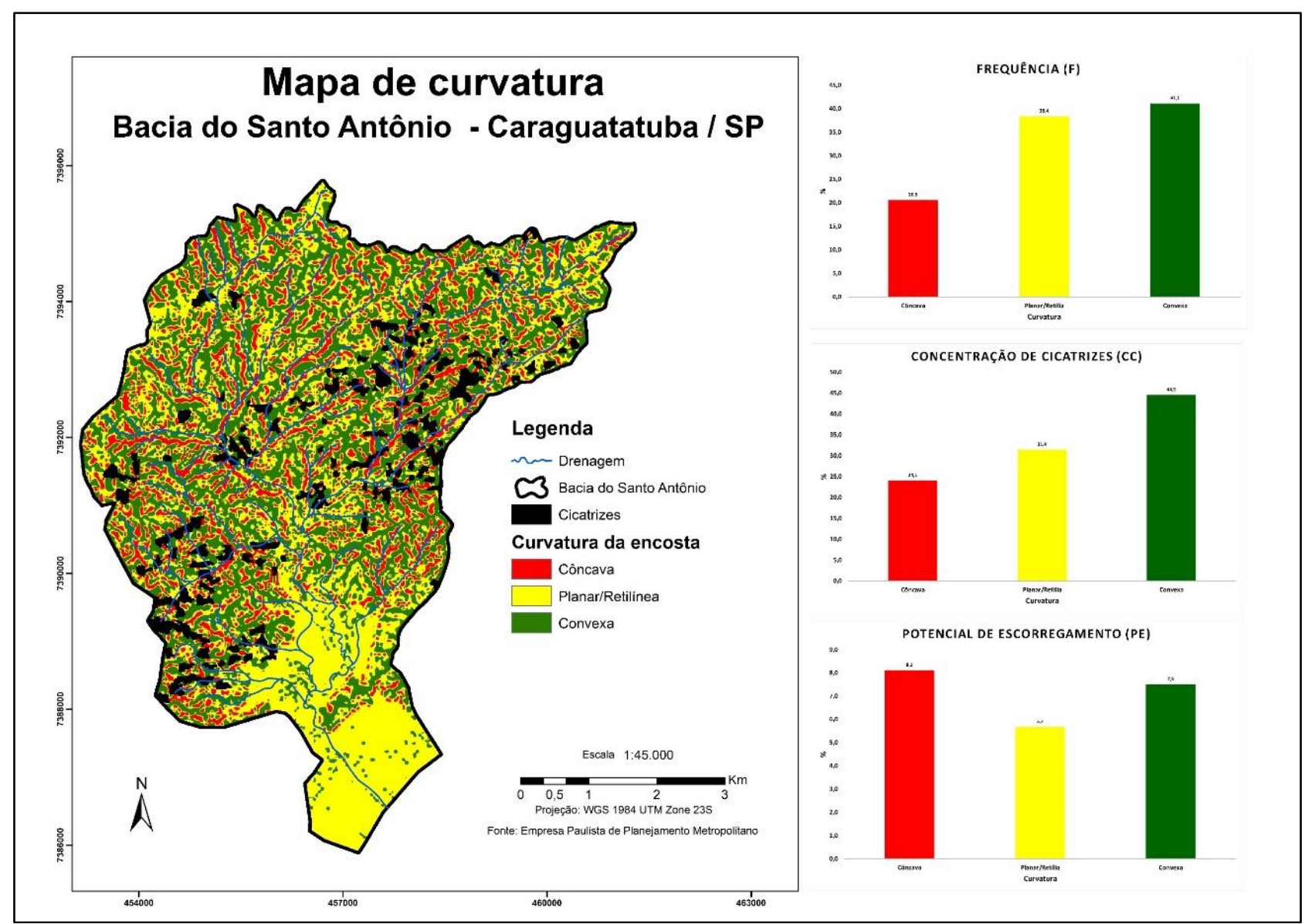

Figura 5: Curvatura na BSA, onde verifica-se maior frequência de encostas convexas na bacia, apesar desta apresentar maior PE para encostas do tipo côncavas.

\section{CONSIDERAÇÕES FINAIS}

Ao realizar uma análise quantitativa por meio dos índices de CC, PE e F, foi possível inferir que as classes preferenciais para ocorrência de escorregamentos na BSA coincidem com os parâmetros citados em alguns trabalhos já realizados na Serra do Mar, o que corrobora os dados obtidos nesta pesquisa que tinha como objetivo compreender a influência de tais parâmetros na distribuição dos escorregamentos na bacia hidrográfica.

A existência de classes morfológicas e geológicas mais suscetíveis aos escorregamentos foi confirmada nesta pesquisa. Embora a maior concentração das cicatrizes tenha sido registrada na porção central da bacia, sustentada pelos granitóides Caraguatatuba, foram os quartzitos que se mostraram mais suscetíveis ao processo, uma vez que estão em uma área intensamente falhada, além da localização em encostas côncavas com altos ângulos de encosta. Isso demonstra que os parâmetros morfológicos não podem ser analisados de maneira independente, devendo sempre ser vistos em conjunto levando sempre em consideração o contexto geológico da área. Tal resultado poderá ser utilizado em futuros mapeamentos de áreas de risco no município, auxiliando no planejamento urbano e reduzindo assim, maiores danos a sociedade.

\section{AGRADECIMENTOS}

Os autores agradecem a Fundação de Amparo à Pesquisa do Estado de São Paulo (FAPESP) pelo apoio financeiro para a realização da pesquisa, ao Programa de Pós-Graduação em Geografia Física (PPGF) da Universidade de São Paulo e aos membros do Grupo de Pesquisa de Processos Morfodinâmicos (GPMorfo). 


\section{REFERÊNCIAS}

ALMEIDA, F.F.M; CARNEIRO, C.D.R. Origem e evolução da Serra do Mar. In: Revista Brasileira de Geociência, v.28 - São Paulo, p.135-150. 1998.

CRUZ, O. A Serra do Mar e o Litoral na Área de Caraguatatuba - SP. Contribuição à Geomorfologia Litorânea Tropical. Tese de Doutorado. IG - Série Teses e Monografias nº 11, 181p. 1974.

FERNANDES, N. F.; AMARAL, C.P. Movimentos de Massa: Uma Abordagem Geológico-Geomorfológica. In: GUERRA, A. J. T. e CUNHA, S. B. (Org.). Geomorfologia e Meio ambiente. Rio de Janeiro: Bertrand Brasil, p. 123- 181. 1996.

FERNANDES, N. F.; GUIMARÃES, R.F.; GOMES, R.A.T.; VIEIRA, B.C.; MONTGOMERY E D.R.; GREENBERG, H. Condicionantes Geomorfológicos dos Deslizamentos nas Encostas: Avaliação de Metodologias e Aplicação de Modelo de Previsão de Áreas Suscetíveis. In: Revista Brasileira de Geomorfologia, V.2; Nº1; p.51-71. 2001.

FERREIRA, F. S. Análise da influência das propriedades físicas do solo na deflagração dos escorregamentos translacionais rasos na Serra do Mar (SP). Dissertação de Mestrado, Universidade de São Paulo, São Paulo, SP. 113p. 2013

FÚlFARO, V.J., PONÇANO, W.L., BISTRICHI, C.H., STEIN, D.P. Escorregamento de Caraguatatuba: expressão atual e registro na coluna sedimentar da planície costeira adjacente. In: Congresso Brasileiro de Geologia de Engenharia, $1^{\circ}$, Rio de Janeiro: Anais Rio de Janeiro, Associação Brasileira de Geologia de Engenharia, v. 2, p.341-346. 1976.

GAO, J. Identification of topographic settings conducive to landsliding from DEM in Nelson County, Virginia, U.SA. Earth Surface Processes and Landforms, v.18, p.579-591. 1993.

HART, M. G. Geomorphology: Pure and Applied. London, Allen \& Unwin, p. 81-107. 2007.

LAN, H. X.; ZHOU, C. H.; WANG, L. J.; ZHANG, H. Y.; LI, R. H. Landslide hazard spatial analysis and prediction using GIS in the Xiaojiang watershed, Yunnan, China. Engineering Geology 76, p. 109-128. 2004.

MONTGOMERY, D.R; DIETRICH, W.E. A physically-based model for topographic control on shallow landsliding. Water Resources Research 30 (4): p. 1153-1171. 1994

NERY, T. D.; VIERA, B. C. Susceptibility to shallow landslides in a drainage basin in the Serra do Mar, São Paulo, Brazil, predicted using the SINMAP mathematical model. Bulletin of Engineering Geology and the Environment (Print), v. 74, p. 369-378. DOI 10.1007/s10064-014-0622-8. 2004.

PACHAURI, A. K.; PANT, M. Landslide hazard mapping based on geological attributes. Engineering Geology 32, p. 81-100. 1992.

SIDLE, R.C; PEARCE, A.J.; O'LOUGHLIN, C.L. Natural factors affecting slope stability. Hillslope Stability and Land Use. Washington: American Geophysical Union, p. 31-72. 1995.

VIEIRA, B.C.; RAMOS, H. A. A. Aplicação do Modelo Shalstab para mapeamento da suscetibilidade a escorregamentos rasos em Caraguatatuba, Serra do Mar (SP). Revista do Departamento de Geografia USP, v.29, p.161 -164. 2015.

VIEIRA, B. C.; FERNANDES, N. F.; AUGUSTO FILHO, O. Shallow landslide prediction in the Serra do Mar, São Paulo, Brazil. Natural Hazards and Earth System Sciences, v. 10, p. 1829-2010. 2010. 